\title{
AS CHUVAS EM CERES - GO (BRASIL): CARACTERÍSTICAS SAZONAIS
}

\section{TAINÁ APARECIDA ALVES SOUZA}

Licenciada em Ciências Biológicas pelo Instituto Federal de Educação, Ciência e Tecnologia

Goiano, Campus Ceres ${ }^{1}$

taina souza2@hotmail.com

THALISSON RAFAEL SOUSA

Licenciado em Ciências Biológicas pelo Instituto Federal de Educação, Ciência e Tecnologia Goiano, Campus Ceres ${ }^{1}$

thalissonrafael22@hotmail.com

\section{NURIA PÉREZ GALLARDO}

Doutora em Ciências da Engenharia Ambiental. Professora da Universidade Federal do Sul e Sudeste do Pará2

nuria_perez@unifesspa.edu.br

\section{ELIS DENER LIMA ALVES}

Doutor em Ciências da Engenharia Ambiental. Professor do Instituto Federal de Educação, Ciência e Tecnologia Goiano, Campus Ceres ${ }^{1}$

\author{
elis.alves@ifgoiano.edu.br
}

RESUMO: Os padrões pluviométricos de uma região podem ter impactos diretos sobre vários setores da sociedade, tais como atividades agroindustriais e socioeconômicas. Dessa forma, o objetivo dessa pesquisa foi analisar a variabilidade temporal das chuvas no município de Ceres, a fim de verificar variações nos padrões da precipitação, bem como compreender sua sazonalidade. Primeiramente, foi realizada aquisição dos dados de chuvas disponibilizados pela Agência Nacional de Águas (ANA) do período de 1972 a 2015, posteriormente, foram realizadas análises da homogeneidade dos dados e falhas na série histórica. Para a análise da variabilidade temporal/sazonal das chuvas em Ceres elaborou-se gráficos dos totais pluviométricos anuais e totais mensais. Posteriormente, elaborou-se gráficos de bloxplots e dendogramas utilizando-se o método de Ward's e a distância Euclidiana como separador, assim como as estatísticas descritivas das chuvas do período estudado. Conclui-se que as chuvas da região de Ceres possuem forte componente sazonal, caracterizado por uma estação seca (abril a setembro) e uma estação chuvosa (outubro a março), característica típica das áreas de cerrado do planalto central. Apesar de apresentar variações pluviométricas durante este período, os desvios elevados foram pontuais.

PALAVRAS-CHAVE: Pluviosidade; Sazonalidade; Clima.

\section{RAINFALL IN CERES - GO (BRAZIL): SEASONAL CHARACTERISTICS}

ABSTRACT: The pluviometric patterns of a given region can directly impact several sectors of society, such as agro-industrial and socio-economic activities. Thus, the objective of the present study was to analyze the temporal variability of rainfall in the municipality of Ceres-GO, Brazil, in order to search for variations in precipitation patterns, as well as understand their seasonality. Initially, the rainfall data provided by the National Water Agency (ANA) from 1972 to 2015 were acquired, after which the homogeneity of the data and gaps in the historical series were analyzed. For the analysis of the temporal/seasonal variability of rainfall in Ceres, graphs of the annual and monthly pluviometric totals were generated. Subsequently, boxplot graphs and dendrograms were drawn up using Ward's method and the Euclidean distance as a separator, as well as the descriptive statistics of the rains during the studied period. It was concluded that the rains in the Ceres region have a strong seasonal component, characterized by a dry season (April to September) and a rainy season (October to March), typical of the Cerrado areas of the Central Plateau. Despite the occurrence of pluviometric variations during this period, high deviations were occasional.

KEYWORDS: Rainfall; Seasonality; Weather.

\footnotetext{
${ }^{1}$ Endereço para correspondência: IF Goiano - Rodovia GO-154, Km 03, s.n., CEP: 76300-000, Ceres-GO.

${ }^{2}$ Endereço para correspondência: UNIFESSPA - Folha 17, Quadra 4, Lote Especial - Nova Marabá, CEP: 68.505-080, Marabá-PA.
}

Tainá A. A. Souza, Thalisson R. Souza, Nuria P. Gallardo, Elis D. L. Alves. As chuvas em Ceres (Brasil): características sazonais Brazilian Geographical Journal: Geosciences and Humanities research medium, Ituiutaba, v. 11, n. 2, p. 170-180, ago./dez. 2020. 


\section{LAS LLUVIAS EN CERES - GO (BRASIL): CARACTERÍSTICAS ESTACIONALES}

RESUMEN: Los índices pluviométricos de una región pueden tener impactos directos sobre varios sectores de la sociedad, como por ejemplo en las actividades agroindustriales e socioeconómicas. De esa forma, el objetivo de este trabajo fue analizar la variabilidad temporal de las lluvias en el municipio de Ceres, con la intención de verificar variaciones en los modelos de precipitación, así como entender su estacionalidad. Primeramente, fue realizada una recopilación de datos de lluvias disponibles en la Agencia Nacional de Aguas (ANA) durante un periodo de tiempo, desde 1972 hasta 2015. Posteriormente se realizaron análisis de homogeneidad de los datos e identificación de los fallos en la serie histórica. Para el análisis de la variabilidad temporal/estacional de las lluvias en Ceres se elaboraron gráficos con los valores pluviométricos totales anuales y totales mensuales. Posteriormente, se realizaron los gráficos de bloxplots y dendogramas utilizando el método Ward's y la distancia Euclediana como separador, además fue utilizada la estadística descriptiva de las lluvias en el periodo estudiado. Este trabajo concluye que las lluvias en la región de Ceres poseen una fuerte componente estacional, caracterizada por una estación seca (de abril a septiembre) y una estación lluviosa (de octubre a marzo), característica típica de las áreas de sabana de la meseta central. A pesar de presentar variaciones pluviométricas durante ese periodo, los desvíos fueron puntuales.

PALABRAS CLAVE: Pluviosidad; Estacional; Clima.

\section{INTRODUÇÃO}

A precipitação é uma das variáveis meteorológicas mais importantes para os estudos climáticos (ALVES, 2011). E são os setores produtivos da sociedade tanto econômico quanto social, que sofrem as influências que esta pode ocasionar quando ocorrida em excesso ou em escassez, resultando em enchentes, assoreamento dos rios, morte de animais, ou até mesmo a falta de água para abastecimento (AMORIM et al., 2008).

Segundo Braz et al. (2007) a sazonalidade e espacialização da chuva interferem na preservação do solo e no planejamento de atividades silvicultoras e agrícolas. A previsão da ocorrência de cheias e a quantificação dos efeitos destas sobre as cidades e culturas agrícolas é de fundamental importância para o planejamento das atividades humanas.

Durante uma precipitação existe grande probabilidade de que a quantidade medida por duas ou mais estações pluviométricas próximas seja diferente, porém com elevada correlação. Portanto, a chuva varia no tempo e no espaço, sendo perceptível que uma mesma quantidade de chuva não cai uniformemente sobre uma determinada área (ALVES; PRADO; SPECIAN, 2011).

A análise dessa distribuição é importante, já que suas variações ao longo dos anos é que determina o clima característico de uma região, sendo relevante este reconhecimento para as atividades humanas que são dependentes socioeconomicamente (MARIN; SENTELHAS e VILLA NOVA, 2000). De acordo com Silva et al. (2007) a precipitação pluvial é um dos elementos meteorológicos de extrema importância, pois além de estar relacionada com as atividades agrícolas, como já mencionado, interferindo diretamente na produção, está também ligada a diversos setores da sociedade, cujas variações, podem afetar além da economia, o meio ambiente e a sociedade em geral.

A região Centro-Oeste possui áreas com elevada variabilidade no regime pluviométrico. Normalmente, o conhecimento dessa variabilidade ocorre por meio das relações de intensidade, frequência e duração das chuvas (BACK, 2009). Considerando-se o fato de não haver estudo sobre variações pluviométricas históricas em Ceres e a importância deste município para a região, bem como os diversos fatores que são influenciados pela chuva, como planejamento urbano, agricultura, e até mesmo cobertura vegetal da região, fazse necessário uma análise dos dados de precipitação, a fim de traçar um perfil deste município, analisando as variações ao logo do tempo. 
A microrregião de Ceres tem na atividade agrícola o elemento fundamental de sua história socioeconômica, relacionada, sobretudo, à ocupação dessa região por meio da expansão da fronteira agrícola (FERREIRA; DEUS, 2011; SILVA; BARBALHO; FRANCO, 2013). Essa microrregião leva o nome de município de Ceres, que se originou em 1942 através do projeto das Colônias Agrícolas Nacionais. A colônia goiana, conhecida pela denominação de CANG, tinha o objetivo principal de atrair agricultores de todas as partes do Brasil para ocupar o interior do país visando à implantação de uma agricultura moderna (SILVA; BARBALHO; FRANCO, 2013).

Por estas razões, o estudo das características das condições climáticas é fundamental para a qualidade de vida, visto que, de acordo com Lucena, Stosic e Cunha Filho (2015) o clima é capaz de influenciar uma população a nível econômico e social, sendo que ao levar em consideração o regime pluviométrico de uma localidade define-se as melhores culturas a serem adotadas para aquela região, podendo ainda influenciar na escolha de locais próprios para habitação e lazer, por exemplo (LONGO; SAMPAIO e SUSZEK, 2006). Considerando todos estes fatores, o objetivo dessa pesquisa foi analisar a variabilidade temporal das chuvas no município de Ceres por meio de serie temporal e levantamento histórico sobre eventos de seca ou chuvas intensas, a fim de verificar variações no padrão de precipitação na região.

\section{MATERIAL E MÉTODOS}

\section{Área de estudo}

O município Ceres está localizado na região do "Vale do São Patrício" e ocupa área de $214,322 \mathrm{~km}^{2}$. A cidade de Ceres (Figura 1) foi fundada com objetivo de atrair agricultores para a região, já que essa apresentava na época condições favoráveis à produção agrícola e ocupação, por possuir solos ricos em minerais essenciais para o cultivo, bem como o fato de estar próxima ao rio das Almas, o qual fornece certa estabilidade para uso dos recursos hídricos (CASTILHO, 2009).

Figura 1 - Localização da cidade de Ceres - Goiás

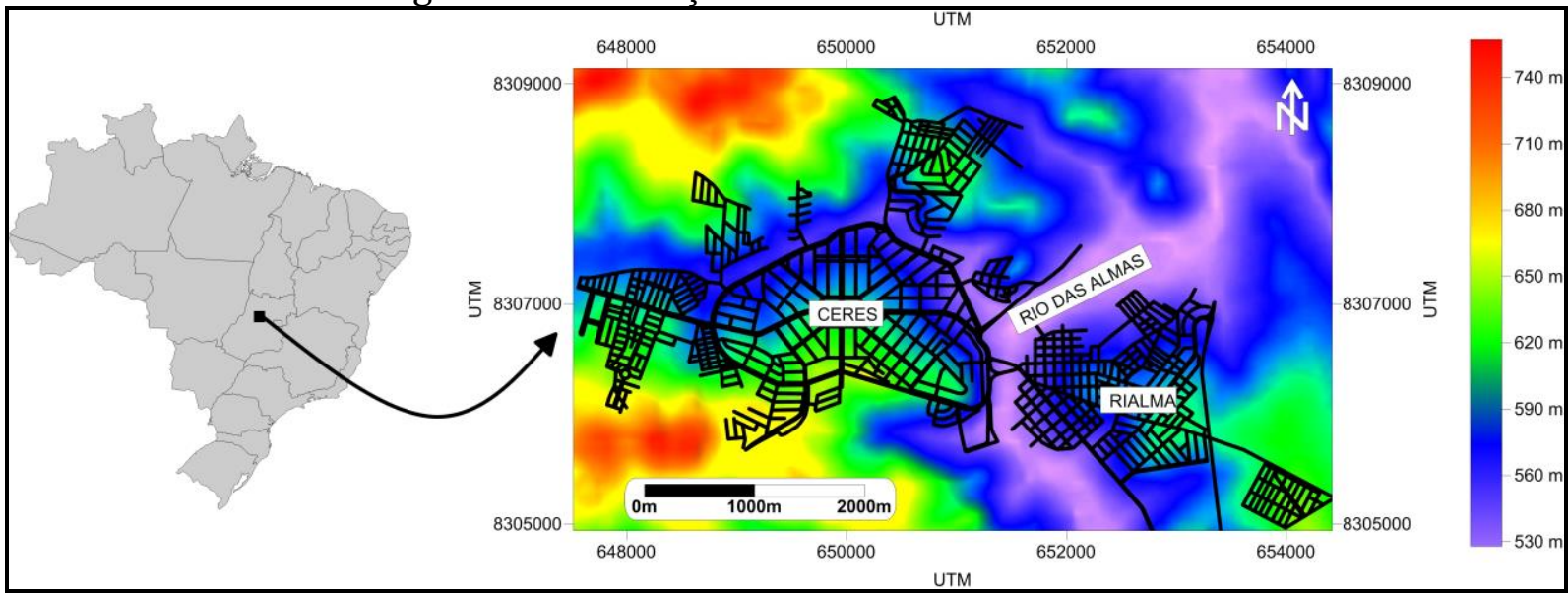

Fonte: Compilação dos autores 3.

A expansão das plantações canavieiras na microrregião de Ceres, em Goiás, é um fenômeno recente. A região experimentou um aumento demográfico significativo a partir das décadas de 1940, quando políticas governamentais foram implantadas para garantir a imigração e o povoamento dessa nova área agrícola, que forneceria matérias primas e alimento para abastecer os grandes centros urbanos em expansão no país. Entretanto, a

${ }^{3}$ Figura elaborada a partir de dados SRTM (Shuttle Radar Topography Mission) e Google Earth (2020).

Tainá A. A. Souza, Thalisson R. Souza, Elis D. L. Alves. As chuvas em Ceres (Brasil): características sazonais Brazilian Geographical Journal: Geosciences and Humanities research medium, Ituiutaba, v. 11, n. 2, p. 170-179, ago./dez. 2020 
partir da década de 1980, foi verificado o deslocamento da produção de alimentos para a produção dos biocombustíveis (SILVA; BARBALHO; FRANCO, 2013).

Com o passar do tempo, profundas alterações econômicas e sociais ocorreram no munícipio de Ceres. Atualmente, possui aproximadamente 22 mil habitantes, segundo o Instituto Brasileiro de Geografia e Estatística - IBGE (2017). Ceres é reconhecida especialmente pelos seus serviços na área da saúde e educação, sendo referência em relação à municípios próximos. O solo da região ainda continua sendo utilizado na produção sucroalcooleira e pastagens por pequenos agricultores. Já na área urbanizada ocorre processos erosivos em decorrência de seu uso, manejo e planejamento inadequado na implantação de projetos de engenharia (MORETI, 2003).

\section{Análise dos dados}

Para atingir os objetivos propostos, que é analisar a variabilidade temporal das chuvas no município de Ceres, a fim de se verificar variações no padrão de precipitação, optou-se por aplicar as seguintes técnicas:

Primeiramente foi realizada aquisição dos dados de chuvas disponibilizados pela Agencia Nacional de Águas (ANA) do período de 1972 a 2015. Os dados foram organizados em planilhas do software Excel. A homogeneidade dos dados foi averiguada por meio do teste de Cochran e não se detectou falhas no registro de dados. Por meio das planilhas foram separados os intervalos de frequência entre os registros de precipitação diária e, após, foram confeccionados os gráficos mensais de frequência. Para a análise da variabilidade temporal/sazonal das chuvas em Ceres elaborou-se gráficos dos totais pluviométricos anuais e totais mensais. Posteriormente, elaborou-se gráficos de bloxplots e dendogramas, utilizando-se do método de Ward's e a distância Euclidiana como separador, assim como as estatísticas descritivas das chuvas do período estudado.

\section{RESULTADOS E DISCUSSÃO}

A partir da análise dos dados relativos as chuvas do período de 1972 a 2015, observouse diversos aspectos inerentes à variabilidade das chuvas em Ceres, tais como: pluviosidade média, precipitação anual e mensal e distribuição da precipitação ao longo do ano.

Quanto à pluviosidade média do período analisado, esta foi de aproximadamente 1475 mm anuais, conforme a figura 2. Dados semelhantes foram obtidos por Lopes et al. (2012), considerando o período de 1999 a 2010 no baixo curso do rio Claro, em Goiás.

A pluviosidade anual foi bastante variável, característica inerente da precipitação, conforme ALVES, 2016; ALVES; PRADO; SPECIAN (2011), apresentando desvio padrão de $262 \mathrm{~mm}$. Dos 44 anos analisados, 25 anos apresentaram pluviosidade inferior à média: 0 ano de 1999 teve o maior desvio negativo em relação à média $(398 \mathrm{~mm})$. Cinco anos $(1976,1978$ e 1990, 1994 e 2009) ficaram em torno da média (-1,1 e -1 mm). 17 apresentaram pluviosidade anual superior à média, com destaque para os anos de 1980 e 1992 com desvios positivos de 619 e 617, respectivamente (Figura 2).

Os totais anuais apresentados (Figura 2) foram distribuídos de forma variável entre os meses do ano, ocasionando a sazonalidade que é bem característica da pluviometria da região dos cerrados. Essa variabilidade sazonal é constituída por duas estações com características pluviométricas dessemelhantes, conforme se pode notar na figura 3. Na estação de seca os níveis de precipitação são menores, com pouca ou nenhuma precipitação, e na estação chuvosa apresenta níveis maiores de precipitação. Quanto à estação seca, caracterizada como inverno seco, esta compreende os meses de abril a setembro, e conta com pluviosidade média de 29,2 mm mensais, sendo bastante variável os valores máximos e mínimos de precipitação para os meses que compõem esta estação, com desvio padrão médio de $26 \mathrm{~mm}$. 


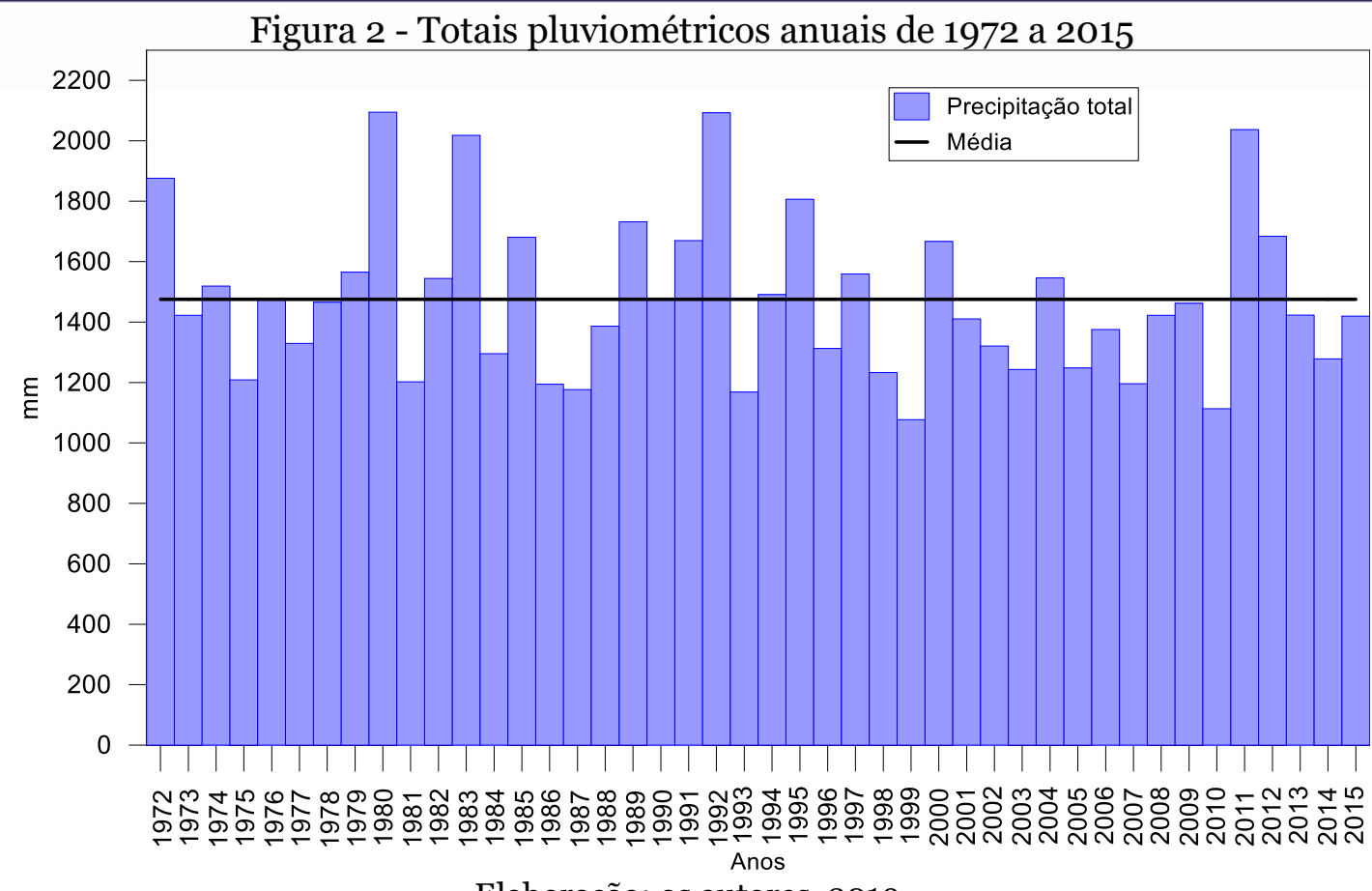

Elaboração: os autores, 2019.

A estação chuvosa, que engloba os meses de outubro a março, compreende níveis maiores de precipitação pluviométrica, alcançando média de $216 \mathrm{~mm}$ mensais, e assim como a estação seca, apresentou variação significativa dos valores máximos e mínimos mensais, característico do verão chuvoso, com desvio padrão médio de 90 mm (Figura 3, 4 e Tabela 1).

Figura 3 - Dendograma dos dados pluviométricos mensais de 1972 a 2015

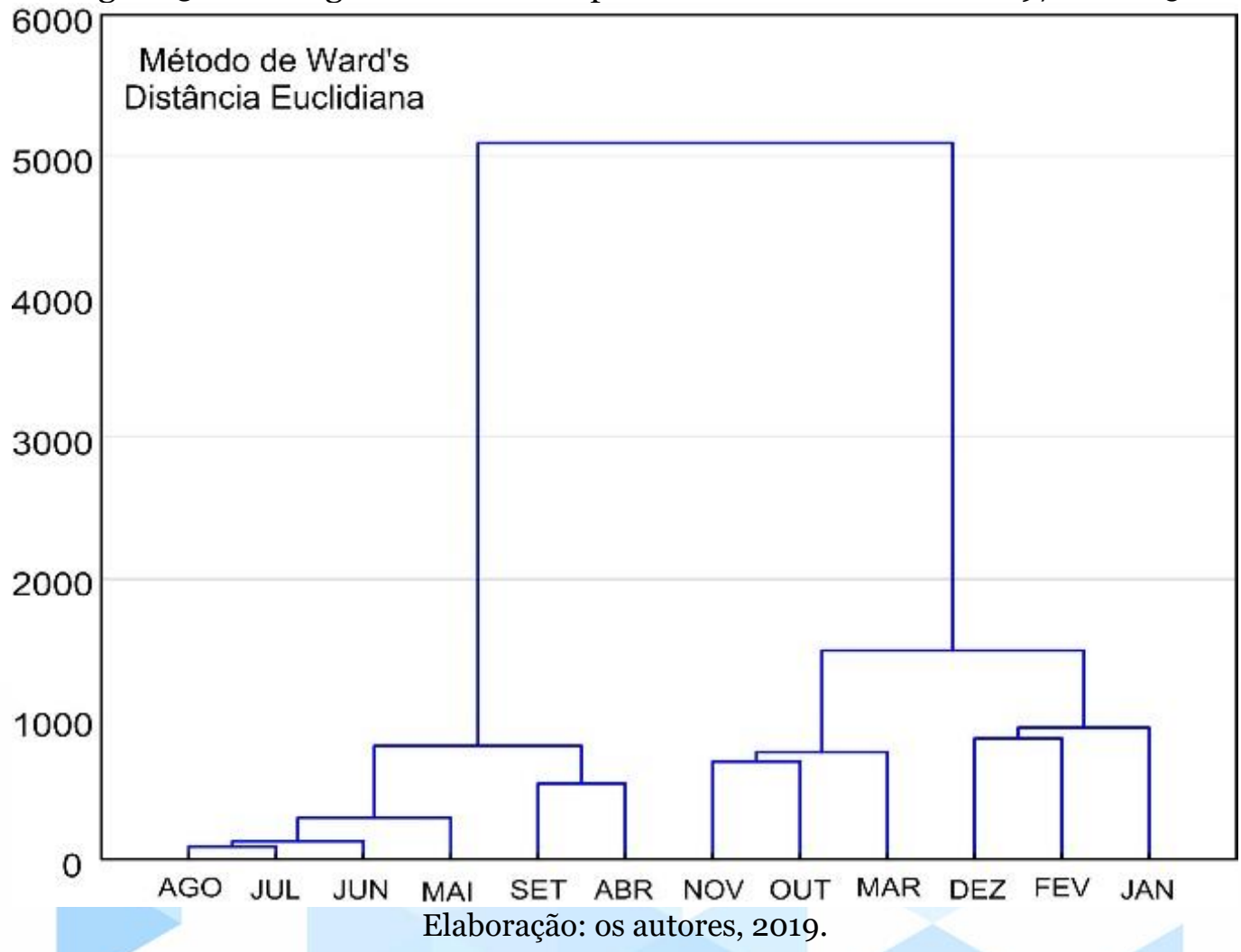

Tainá A. A. Souza, Thalisson R. Souza, Elis D. L. Alves. As chuvas em Ceres (Brasil): características sazonais Brazilian Geographical Journal: Geosciences and Humanities research medium, Ituiutaba, v. 11, n. 2, p. 170-179, ago./dez. 2020 


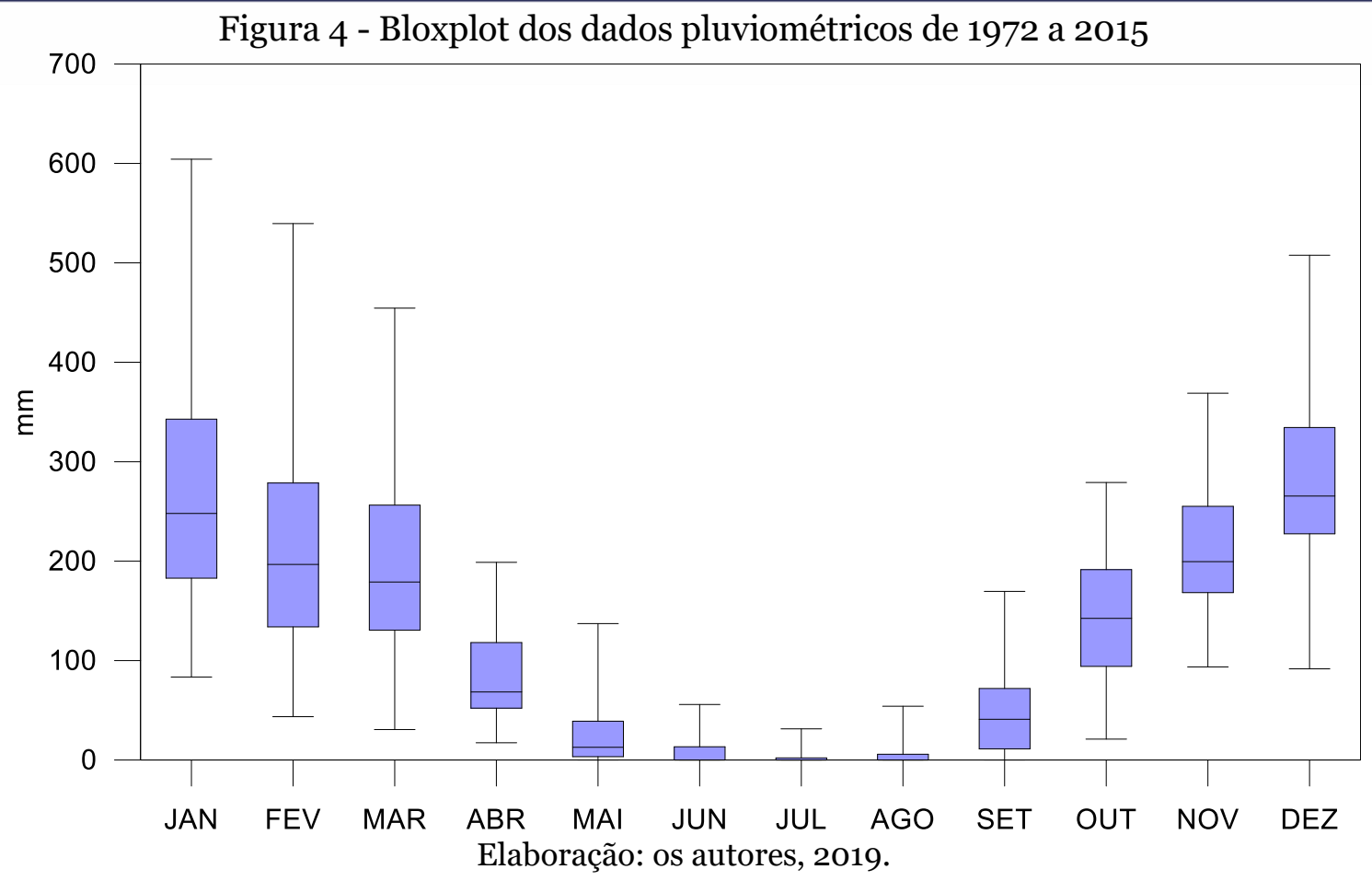

Esta sazonalidade influencia na agricultura da região, sendo responsável por definir os períodos de plantio e safra da cana de açúcar e outras culturas que se desenvolvem em torno da cidade de Ceres.

A espacialização dos totais mensais da precipitação (Figura 5) deixa evidente os padrões sazonais da precipitação, confirmando as duas estações, seca e chuvosa, observadas pelo agrupamento da figura 3. O período seco fica evidente nos tons avermelhados e o período chuvoso dos tons amarelos aos azuis, entretanto, nota-se que dentro das estações podem ocorrer variabilidade intrasazonal devido as questões climáticas regionais e globais, como é o caso, por exemplo, do El Niño. Marcuzzo e Romero (2013) observaram que a maior e a menor precipitação máxima diária anual, registrada para o estado de Goiás no período de 1977 a 2006, foi de 461,3 mm (1997) e 360,3 mm (2003), respectivamente, e ocorreram em anos do El Niño moderado $\left(1,3^{\circ} \mathrm{C}\right)$ e fraco $\left(0,5^{\circ} \mathrm{C}\right)$.

Figura 5 - Totais pluviométricos mensais de 1972 a 2015

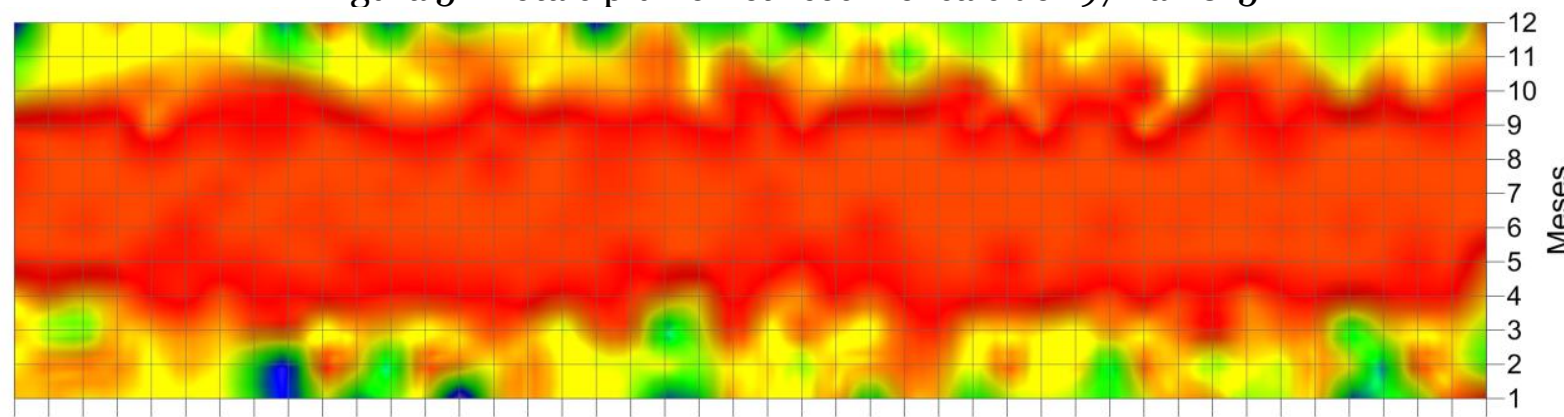

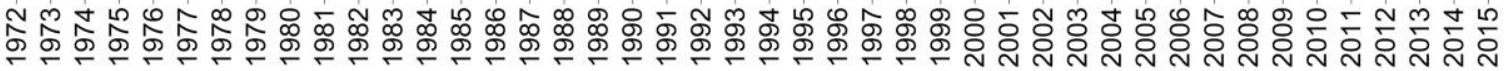
Anos $\mathrm{mm}$

$0 \quad 50100150200250300350400450500550$ Elaboração: os autores, 2019.

Tainá A. A. Souza, Thalisson R. Souza, Elis D. L. Alves. As chuvas em Ceres (Brasil): características sazonais Brazilian Geographical Journal: Geosciences and Humanities research medium, Ituiutaba, v. 11, n. 2, p. 170-179, ago./dez. 2020 Página | 175 
As precipitações máximas e mínimas mensais para este período seco vão desde meses sem precipitação (de maio a setembro) a meses em que a precipitação atingiu 198,8 mm (em abril), como se pode observar na tabela 1. Para o período chuvoso, a precipitação mínima encontrada foi de $21 \mathrm{~mm}$ e máxima de 604,3 mm, nos meses de outubro e janeiro, respectivamente.

Tabela 1 - Estatística básica dos dados pluviométricos de 1972 a 2015

\begin{tabular}{cccccc}
\hline Mês & Mínima & Média & Máxima & Mediana & Desvio Padrão \\
\hline Janeiro & 83,4 & 263,9 & 604,3 & 248 & 112,1 \\
Fevereiro & 43,6 & 215,5 & 539,6 & 196,7 & 110,8 \\
Março & 30,7 & 192,1 & 454,4 & 179,0 & 91,4 \\
Abril & 17,4 & 85,4 & 198,8 & 68,5 & 49,0 \\
Maio & 0 & 23,9 & 137,2 & 12,8 & 28,4 \\
Junho & $\mathrm{O}$ & 7,6 & 55,8 & 0 & 13,5 \\
Julho & $\mathrm{O}$ & 3,6 & 31,3 & $\mathrm{O}$ & 8 \\
Agosto & $\mathrm{O}$ & 6,8 & 54,0 & 0 & 12,7 \\
Setembro & $\mathrm{O}$ & 47,7 & 169,6 & 40,9 & 44,1 \\
Outubro & 21,0 & 140,1 & 279,2 & 142,4 & 62,3 \\
Novembro & 93,6 & 209,5 & 368,9 & 199,5 & 65,3 \\
Dezembro & 91,7 & 278,1 & 507,7 & 265,5 & 97,3 \\
\hline
\end{tabular}

Elaboração: os autores, 2019.

Em sua grande maioria, o total mensal de chuvas para o mês de janeiro ficou entre 200 e 300 mm (Figura 6). Para os meses de fevereiro e março, essa dominância foi de 100 a $200 \mathrm{~mm}$ mensais. Os meses de abril a setembro, que compreendem o período de seca, raramente apresentaram precipitação superior a $100 \mathrm{~mm}$. Para os meses de outubro e novembro, a frequência maior das chuvas esteve também entre 100 a $200 \mathrm{~mm}$ mensais, enquanto o mês de dezembro reflete a frequência encontrada para o mês de janeiro. Estes dados reforçam a existência de períodos com características semelhantes, bem como permite observar uma variação na precipitação anual de cada mês.

Figura 6 - Frequência de classes de precipitação mensal de 1972 a 2015
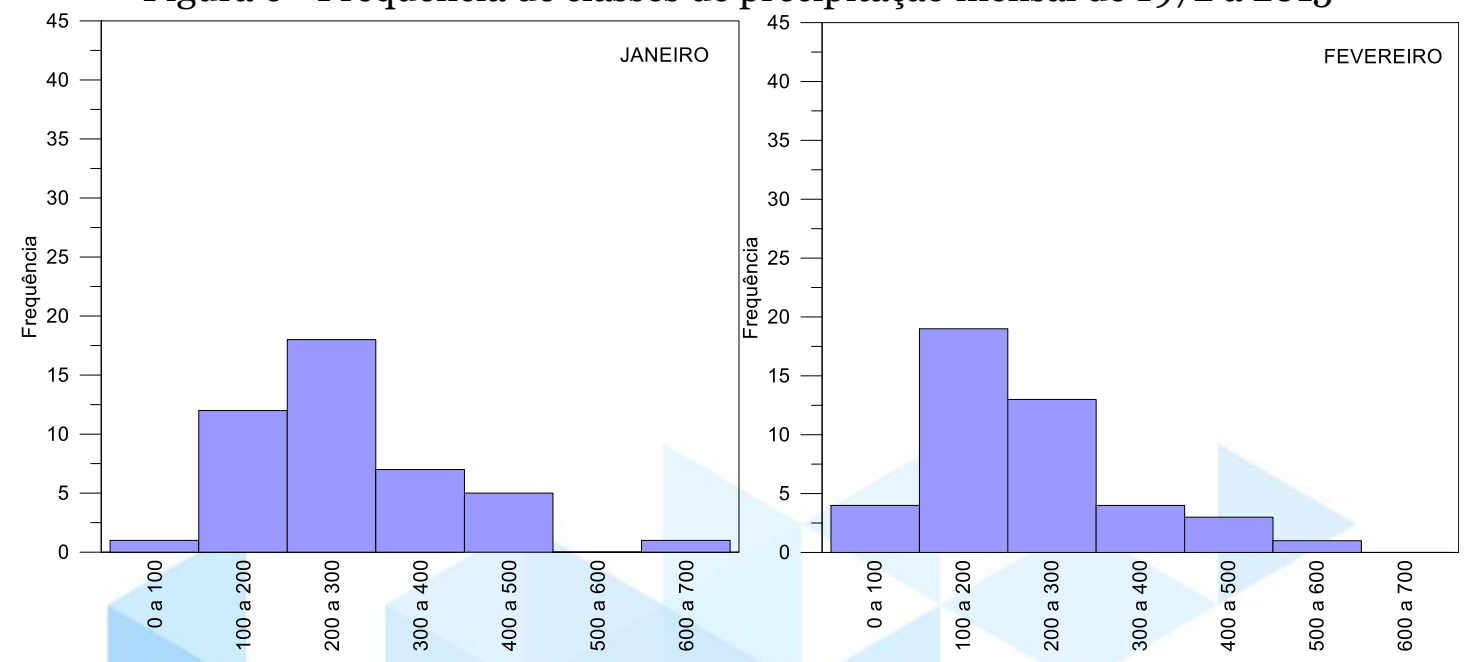

Tainá A. A. Souza, Thalisson R. Souza, Elis D. L. Alves. As chuvas em Ceres (Brasil): características sazonais Brazilian Geographical Journal: Geosciences and Humanities research medium, Ituiutaba, v. 11, n. 2, p. 170-179, ago./dez. 2020 

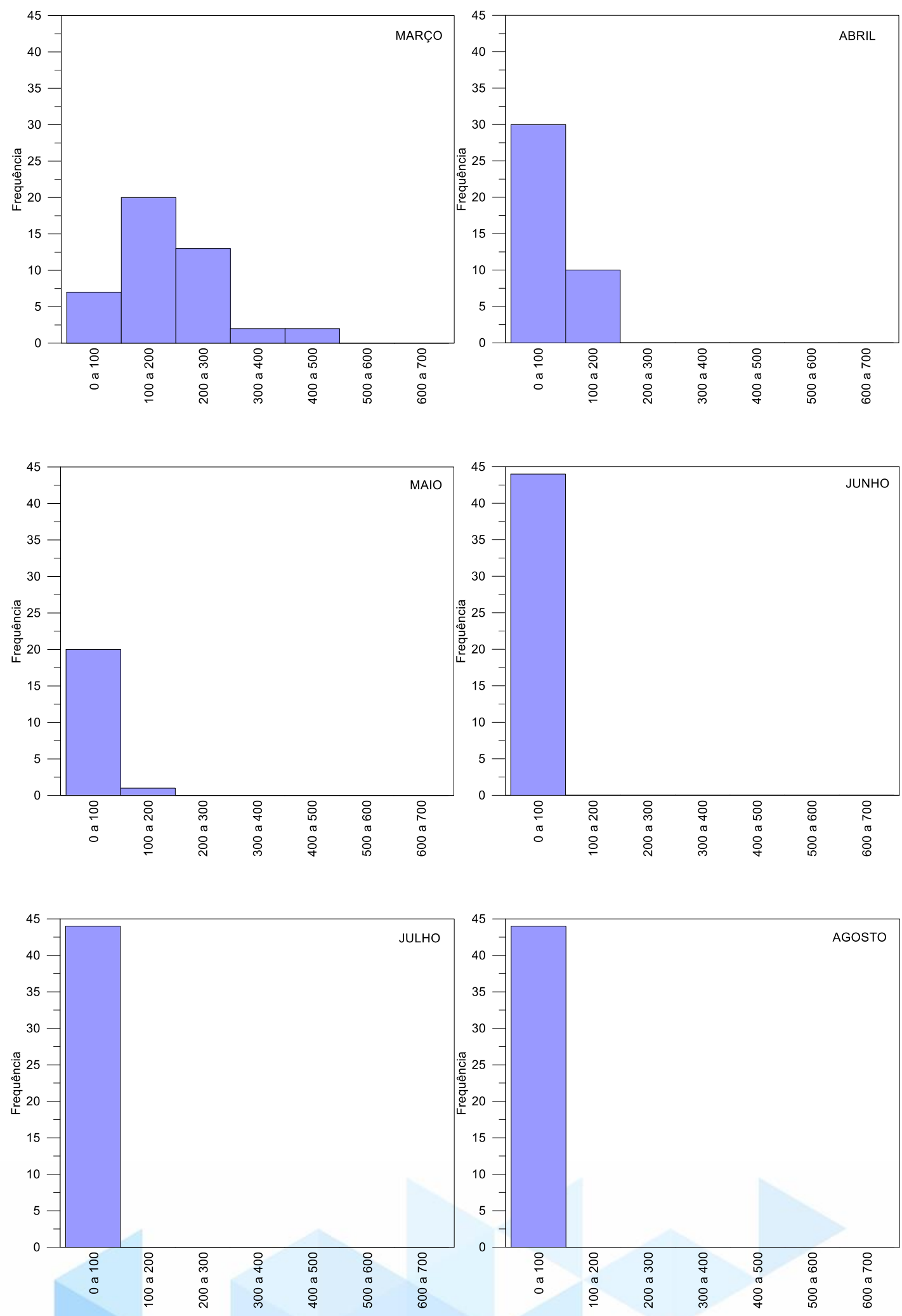

Tainá A. A. Souza, Thalisson R. Souza, Elis D. L. Alves. As chuvas em Ceres (Brasil): características sazonais Brazilian Geographical Journal: Geosciences and Humanities research medium, Ituiutaba, v. 11, n. 2, p. 170-179, ago./dez. 2020 

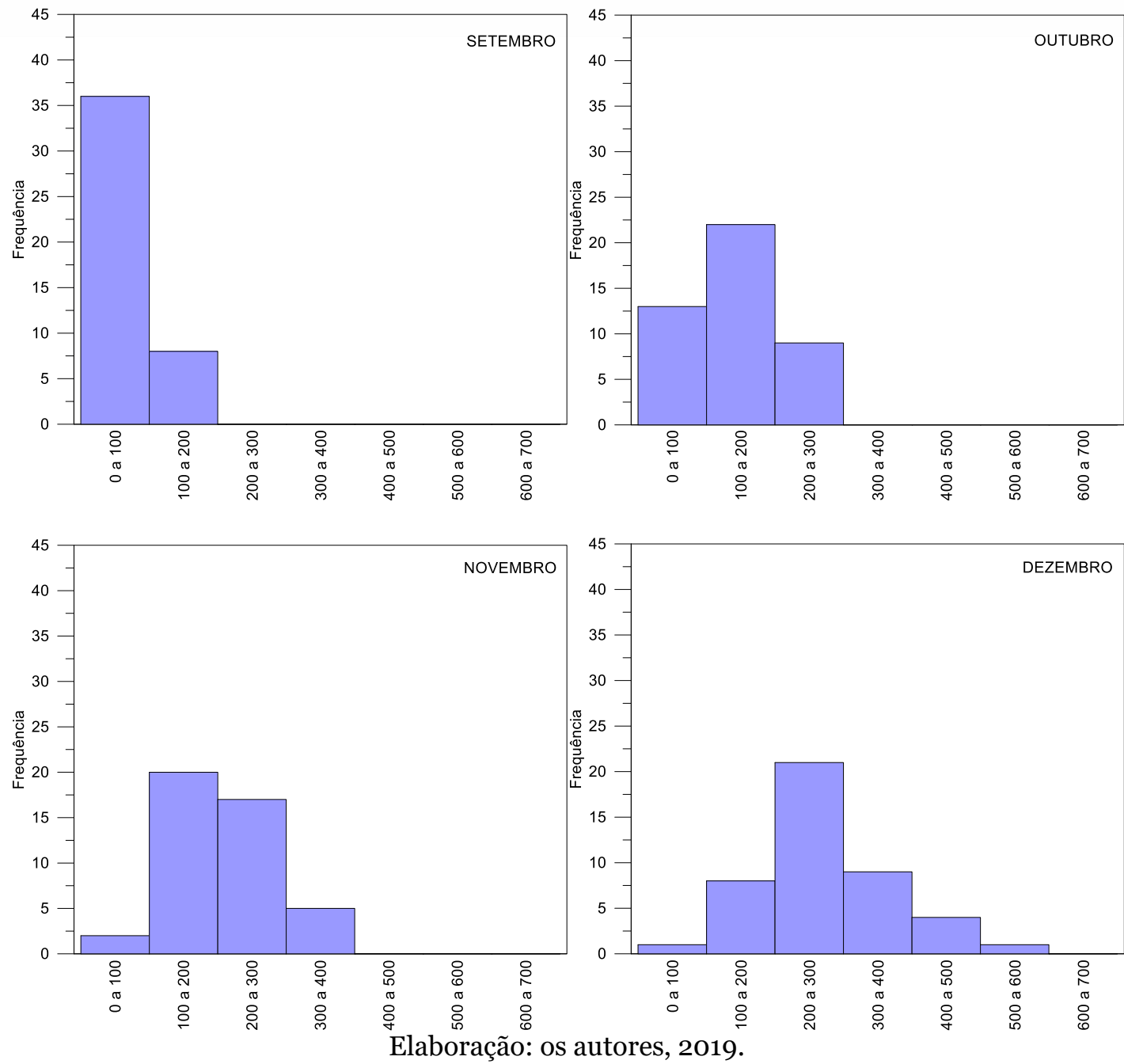

Em levantamento realizado sobre os aspectos históricos da região, não se constatou indícios de eventos de seca ou chuva intensos, que causaram prejuízos significativos à atividade agroindustrial ou de ordem econômica. O clima da região contribui para manter estas atividades em funcionamento, visto que grande parte da agropecuária da região depende de irrigação proveniente do Rio das Almas e seus afluentes, os quais tem seu nível regulado pelas chuvas que precipitam na bacia hidrográfica desse rio. Além disso, o fenômeno de sazonalidade determina quais períodos serão utilizados para plantio e colheita de lavouras.

\section{CONSIDERAÇÕES FINAIS}

Conclui-se que as chuvas da região de Ceres possuem forte componente sazonal, caracterizada por uma estação seca (abril a setembro) e uma estação chuvosa (outubro a março), característica típica das áreas de cerrado do planalto central. Apesar de apresentar variações pluviométricas durante este período, os desvios elevados foram pontuais, o que leva a concluir que os padrões pluviométricos não sofreram mudanças expressivas durante o período analisado. 


\section{REFERÊNCIAS}

LONGO, A. J.; SAMPAIO, S. C.; SUSZEK, M. Equação de chuvas intensas e precipitação provável para o município de Cascavel, PR. Revista Varia Scientia. v. 06, n. 11, p. 119-127, 2006.

LOPES, M. R. et al. Variabilidade espacial e temporal das chuvas no baixo curso do Rio Claro-GO, no período de 1977 a 2010. Revista Geonorte, Edição Especial, V.2, N.4, p.1126 $-1137,2012$.

LUCENA, L. R. R.; STOSIC, T.; CUNHA FILHO, M. Avaliação da precipitação diária do estado de Sergipe utilizando análise de lacunaridade. Rev. Bras. Biom., São Paulo, v.33, n.2, p.268-276, 2015.

MARCUZZO, F. F. N., ROMERO, V. "Influência do el niño e la niña na precipitação máxima diária do estado de Goiás", Revista Brasileira de Meteorologia, v. 28, n. 24, p. 429-440, 2013. .

MARIN, F. R.; SENTELHAS, P. C.; VILLA NOVA, N. A. Influência dos fenômenos El Niño e La Niña no clima de Piracicaba, SP. Revista Brasileira de Meteorologia, v.15, n.1, 123129, 2000.

MORETI, D. et al. Importantes características de chuva para a conservação do solo e da água no município de São Manuel (SP). R. Bras. Ci. Solo, v. 27, p. 713-725, 2003.

SILVA, S. D. E; BARBALHO, M. G. DA S.; FRANCO, J. L. DE A. A expansão sucroalcooleira e a devastação ambiental nas matas de São Patrício, microrregião de Ceres, Goiás. História, histórias, v. 1, n. 2, p. 230-247, 2013.

SILVA, J. C. et al. Análise de distribuição de chuva para Santa Maria, RS. Revista Brasileira de Engenharia Agrícola e Ambiental. v.11, n.1, p.67-72, 2007.

Recebido em: 29/09/2020. Aprovado para publicação em: 28/12/2020. 